\title{
Avaliação do torque para inserção, remoção e fratura de diferentes mini-implantes ortodônticos
}

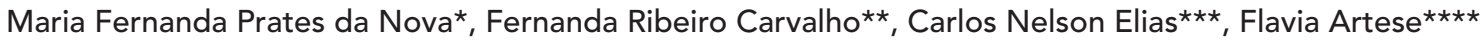

\section{Resumo}

Objetivo: avaliar, em mini-implantes de diferentes dimensões, os seguintes fatores: (a) torque de inserção, (b) torque de remoção, (c) torque de fratura, (d) tensão cisalhante, (e) tensão normal e (f) tipo de fratura. Metodologia: foram utilizados 20 mini-implantes autoperfurantes, 10 da marca SIN e 10 da Neodent com, respectivamente, 8 e $7 \mathrm{~mm}$ de comprimento, todos com 1,6mm de diâmetro. Dos 10 mini-implantes de cada marca, 5 não possuíam perfil transmucoso e 5 tinham perfil de $2 \mathrm{~mm}$, formando 4 grupos: SIN sem perfil (SSP), SIN com perfil (SCP), Neodent sem perfil (NSP) e Neodent com perfil (NCP). Todos os mini-implantes foram inseridos em cortical óssea e removidos com micromotor acoplado a um torquímetro. Os mini-implantes foram, também, submetidos ao ensaio de fratura. Os torques de inserção, remoção e fratura, assim como a tensão cisalhante e normal calculadas, foram comparados entre todos os grupos pela ANOVA. O tipo de fratura foi avaliado em microscópio eletrônico de varredura. Resultados: verificou-se que o grupo NCP apresentou torque de inserção significativamente maior que os demais grupos, porém todos fraturaram durante a inserção ( $\mathrm{n}=$ 2) ou remoção $(n=3)$. Não houve diferença entre os grupos para o torque de remoção. Para o grupo NSP, o torque de fratura foi significativamente menor do que todos os outros grupos. Todos os mini-implantes sofreram fratura do tipo dúctil. Conclusão: uma vez que não houve diferença na resistência mecânica de ambas as marcas, variando apenas a forma, conclui-se que a resistência à fratura pode ser afetada por esta variável.

Palavras-chave: Implantes dentários. Resistência de materiais. Torque. Procedimentos de ancoragem ortodôntica.

\section{INTRODUÇÃO}

A ancoragem ortodôntica é definida como resistência ao movimento dentário indesejável ${ }^{20}$. Tradicionalmente, grupos de dentes são utilizados como unidades de ancoragem ${ }^{1}$, mas podem se deslocar como conseqüência indesejada da força reacional à força aplicada. Aparelhos que necessitam da colaboração do paciente também podem ser usados como mecanismos de ancoragem ${ }^{13,24}$. A ausência de dentes posteriores também pode dificultar a ancoragem adequada.

Com o advento da osteointegração, métodos de

* Mestre em Ortodontia pela UERJ.

** Aluna de especialização em Ortodontia da UERJ.

*** Professor adjunto do Instituto Militar de Engenharia do Rio de Janeiro.

**** Professora adjunta de Ortodontia da UERJ. 
tratamento ortodôntico com controle máximo de ancoragem foram propostos, principalmente para adultos $^{21,22}$. A utilização de implantes osteointegrados, como mecanismo de ancoragem absoluta ${ }^{7}$, foi indicada para o tratamento de casos mais complexos, para a otimização de resultados com mecânicas mais simples ou, ainda, para a diminuição do tempo de tratamento. No entanto, implantes osteointegrados convencionais só podem ser inseridos em locais específicos, como no espaço retromolar ou em áreas edêntulas ${ }^{10,21}$.

A partir de parafusos para estabilização cirúrgica, mini-implantes ortodônticos foram desenvolvidos e utilizados como mecanismos de ancoragem absoluta ${ }^{12}$. Além de serem um recurso de ancoragem eficaz, são de fácil instalação e remoção, e suficientemente pequenos para colocação em diversas áreas do osso alveolar, inclusive no espaço inter-radicular. Estas características fizeram com que os mini-implantes fossem rapidamente aceitos e utilizados clinicamente $e^{4,19}$.

Ao contrário dos implantes dentários osteointegráveis, que são fabricados em titânio puro, os mini-implantes são fabricados com a liga Ti-6AL$4 \mathrm{~V}$ por três razões: (a) os mini-implantes têm diâmetro reduzido e essa liga de titânio tem maior resistência mecânica do que o titânio comercialmente puro; (b) a utilização desses sistemas é baseada na estabilidade mecânica primária, e não na estabilidade secundária, advinda da osteointegração; e (c) o mini-implante deve apresentar facilidade de remoção. Empregando-se a liga Ti-6AL$4 \mathrm{~V}$, que possui características bioativas inferiores ao titânio comercialmente puro, o grau de osteointegração é baixo 9 .

A característica do mini-implante mais importante para a Ortodontia é a estabilidade mecânica, conseguida através da estabilidade primária, que é definida como aquela obtida imediatamente após a inserção. A densidade óssea da área de inserção do dispositivo, a forma e a espessura do mini-implante e a preparação do local em que será inserido possuem um grande impacto na estabi- lidade primária dos mini-implantes. Dependendo do sítio de inserção e da qualidade óssea do local, o ortodontista pode escolher a combinação entre tipo, diâmetro e comprimento do mini-implante mais indicado para aquela região $0^{25}$.

A forma do mini-implante deve prover ancoragem mecânica através da superfície de contato osso/implante e tem que permitir a distribuição de cargas de maneira que não prejudique a fisiologia óssea. A forma (design) do mini-implante também deve limitar o trauma apenas ao momento da inserção e permitir estabilidade primária ${ }^{10}$.

O torque para inserção de um mini-implante traduz a quantidade de estabilidade primária conseguida e é, portanto, um fator importante para o sucesso do mecanismo de ancoragem ${ }^{25}$. Friberg et al. ${ }^{11}$ relataram uma correlação positiva, estatisticamente significativa, entre o torque de inserção do mini-implante e os valores de densidade óssea do local a ser utilizado, e concluíram que métodos utilizados para a medição de torque durante a inserção do mini-implante devem ser usados rotineiramente.

Após a utilização do mini-implante para o tratamento desejado, sua remoção é necessária. Existem poucos estudos avaliando o torque máximo de remoção. Geralmente, os torques de remoção, em estudos de curto prazo, são inferiores aos torques de inserção ${ }^{9,16}$. No entanto, quando existe um período de acompanhamento de mais de 4 semanas, os torques de remoção aumentam significativamente ${ }^{5,6,8}$.

A fratura é um dos fatores de riscos e complicações que ocorrem na utilização dos mini-implantes. Ocorre, normalmente, durante a inserção ou remoção do mini-implante no osso, mas também pode incidir durante a aplicação de força no tratamento ortodôntico. No entanto, a qualidade e a densidade óssea podem influenciar na resistência ao torque de inserção, que, aliada à subperfuração, pode potencializar a fratura da região próxima à cabeça do mini-implante ${ }^{14}$.

Melsen ${ }^{15}$ associou o menor diâmetro do mini- 
implante à maior possibilidade de fratura. Utilizando técnicas para medir a distribuição de estresse, observou que a possibilidade de fratura é maior durante o processo de remoção do que de inserção. As fraturas, geralmente, ocorrem perto do pescoço do mini-implante e a presença de orifícios pode enfraquecer ainda mais o dispositivo.

Buscando maior eficiência, vários tipos e formas de mini-implantes foram lançados no mercado por diferentes fabricantes. Sabe-se que a seleção do diâmetro e do comprimento dos mini-implantes é fator importante para a sua adequada utilização, apesar de poderem ser instalados em diversos sítios da cavidade bucal. No entanto, não existe um protocolo que indique qual tipo de mini-implante é o mais recomendado para cada situação ${ }^{2,3}$. Apesar da literatura ser rica em casos clínicos tratados com mini-implantes, ainda existem dúvidas sobre como as características morfológicas desses dispositivos podem afetar suas propriedades físicas ${ }^{25}$. Desta forma, este trabalho objetivou avaliar, em mini-implantes de fabricantes e dimensões diferentes, o torque de inserção e de remoção, assim como as características mecânicas da fratura sob torção.

\section{MATERIAL E MÉTODOS \\ Amostras de mini-implantes}

Foram utilizados 20 mini-implantes comerciais autoperfurantes, sendo 10 da empresa SIN (Sistema de Implantes Nacional, São Paulo/SP, Brasil) e 10 da empresa Neodent (Curitiba/PR, Brasil). Todos os mini-implantes possuíam 1,6mm de diâmetro, sendo que os da SIN possuíam comprimento de $8 \mathrm{~mm}$ (Fig. 1A, C) e os da Neodent $7 \mathrm{~mm}$ (Fig. 1B, D). Para compor os grupos, foram utilizados cinco mini-implantes sem perfil transmucoso e cinco mini-implantes com perfil transmucoso de cada fabricante (Fig. 1). A amostra foi assim dividida em quatro grupos, que foram denominados: SIN sem perfil (SSP); SIN com perfil (SCP); Neodent sem perfil (NSP) e Neodent com perfil (NCP).

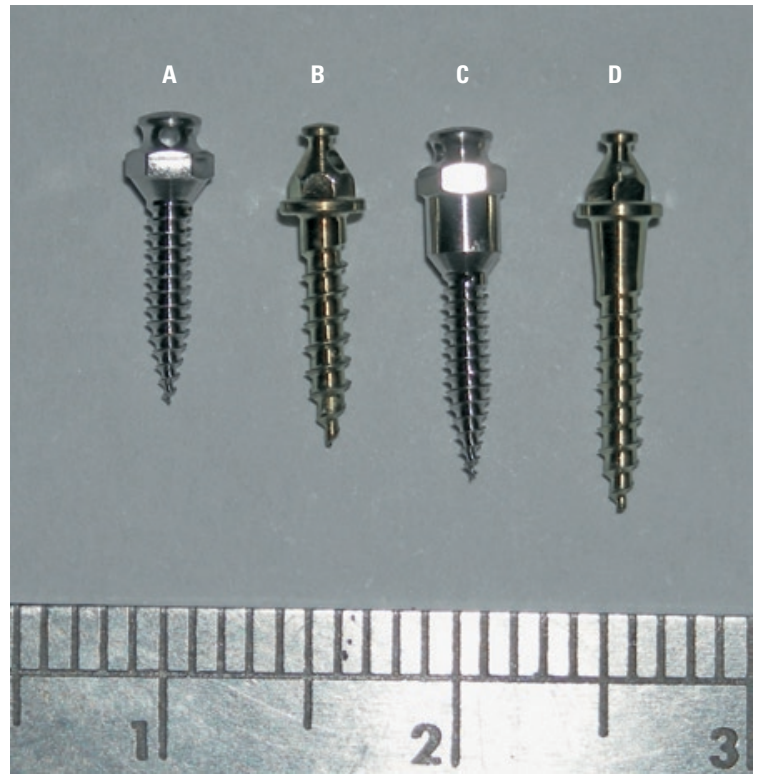

FIGURA 1 - Mini-implantes autoperfurantes, com diâmetro de 1,6mm, que constituíram a amostra: A) mini-implante da Neodent, medindo $7 \mathrm{~mm}$ de comprimento, sem perfil transmucoso (grupo NSP); B) mini-implante da SIN medindo $8 \mathrm{~mm}$ de comprimento, sem perfil transmucoso (grupo SSP); C) mini-implante da Neodent, medindo $7 \mathrm{~mm}$ de comprimento, com perfil transmucoso de $2 \mathrm{~mm}$ (grupo NCP) e D) mini-implante da SIN medindo $8 \mathrm{~mm}$ de comprimento com perfil transmucoso de $2 \mathrm{~mm}$ (grupo SCP)

Todos os procedimentos e ensaios foram realizados nas dependências do Laboratório de Biomateriais do Curso de Engenharia de Materiais do Instituto Militar de Engenharia do Rio de Janeiro (IME).

\section{Preparo das peças ósseas}

Duas tíbias de boi foram obtidas em abatedouro. Estas foram cortadas transversalmente ao seu longo eixo, em segmentos de $15 \mathrm{~mm}$. Retirou-se a medula e a cortical óssea foi mensurada. Os segmentos que possuíam, no mínimo, $9 \mathrm{~mm}$ de espessura foram selecionados e novamente recortados em peças quadrangulares com $10 \mathrm{~mm}$ por lado. Estas dimensões permitiam o posicionamento adequado da peça óssea no torquímetro e asseguravam a inserção completa da broca para perfuração (Fig. 2) e dos mini-implantes a serem avaliados em cortical óssea. Obteve-se, desta forma 20 


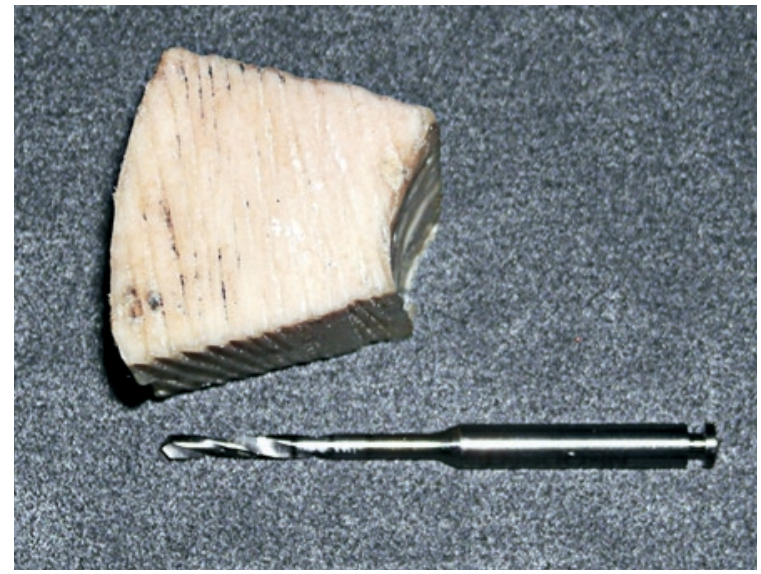

FIGURA 2 - Fragmento de tíbia bovina, recortado em peça com 10mm de largura e comprimento, e $9 \mathrm{~mm}$ de profundidade, para garantir a inserção completa da broca para perfuração e também dos mini-implantes avaliados.

fragmentos ósseos, um para cada mini-implante, que foram mantidos a $4^{\circ} \mathrm{C}$ por três dias, até a data dos ensaios.

Esses fragmentos ósseos foram, então, encaixados em peça metálica regulável para o seu tamanho e forma. Essa peça ficava acoplada a um torquímetro (Lutron torquimeter TQ- 8800, Taipei, Taiwan) preso a um torno de bancada, que impedia qualquer movimentação do mesmo durante o experimento (Fig. 3).

Foi utilizado um motor cirúrgico MC - 101, Omega.02 da Dentscler (Ribeirão Preto/SP, Brasil) ligado a um contra-ângulo redutor 20:1, com 40.000rpm (Anthogyr Instruments, Saclanches, França), para realizar os ensaios de inserção e remoção dos mini-implantes.

Para permitir a inserção dos mini-implantes apenas em cortical óssea, foi realizada uma perfuração no centro da peça óssea. Utilizou-se uma broca de aço cilíndrica com diâmetro de $1,3 \mathrm{~mm}$, específica para perfuração de osso (Neodent Curitiba/PR, Brasil). Esta foi colocada no contra-ângulo e a perfuração foi realizada sob irrigação manual com água.

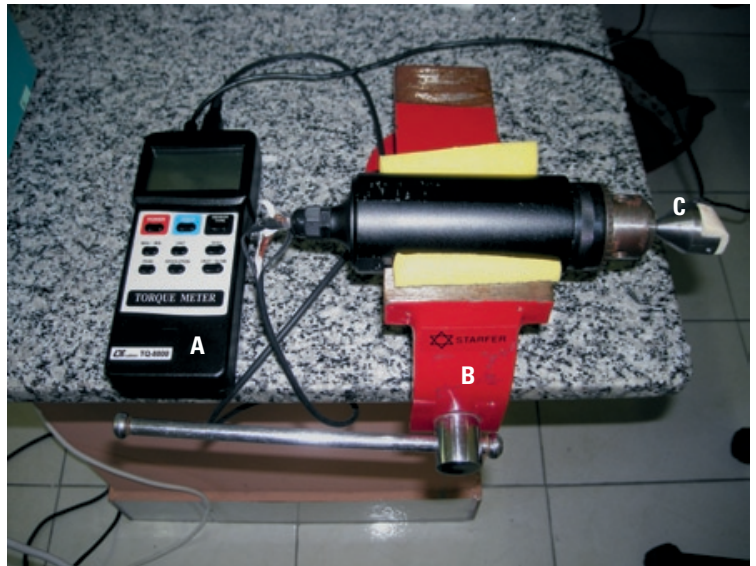

FIGURA 3 - Sistema utilizado para medir o torque de inserção e de remoção dos mini-implantes, constituído por um torquímetro digital (A) preso ao torno de bancada (B), com peça óssea, onde os mini-implantes foram inseridos, presa por posicionador metálico (C).

\section{Ensaios mecânicos}

Em seguida, foi feita a instalação do mini-implante com chave de inserção acoplada ao contraângulo, também sob irrigação manual. Este procedimento de inserção era interrompido quando ocorria o travamento do mini-implante no osso, impedindo o giro do motor. Utilizava-se, então, a chave de torque manual até a inserção completa do mini-implante no osso, i.é., até não se visualizar as roscas do mini-implante. Os mini-implantes foram removidos do osso com o mesmo motor, utilizando-se a opção de rotação reversa, sem necessidade de uso da chave manual.

Durante os ensaios de inserção e remoção, o torque foi medido de maneira contínua. Estes dados foram registrados através da conexão do torquímetro ao computador, que enviava os dados para o programa Lutron 101, versão V0011TW (Lutron Electronic Enterprise, Taipei, Taiwan). Os valores obtidos foram copiados para o Origin PRO 7.0 (Origin Lab Corporation, Northampton, MA, EUA) para a obtenção dos gráficos. Os valores de torques máximos de inserção e de remoção foram obtidos pelos picos máximos dos gráficos. 
Durante os ensaios, tanto de inserção quanto de remoção, alguns mini-implantes sofreram fratura. Os mini-implantes que não fraturaram durante estes ensaios foram submetidos ao ensaio mecânico de fratura à torção, utilizando um dispositivo de mandris acoplado à máquina universal de ensaios mecânicos (EMIC, Curitiba/PR, Brasil) com célula de carga de $500 \mathrm{~N}$.

Para a fratura sob torção, o mini-implante foi preso por mandris nas suas duas extremidades. Um destes mandris é fixo, onde foi presa a ponta do mini-implante. $\mathrm{O}$ outro gira por tração de um fio de polímero preso ao eixo do mandril e à celula de carga, onde se prendeu a cabeça do mini-implante. Com uma extremidade fixa e a outra sofrendo rotação, gera-se uma força de torque sobre o mini-implante, que é registrada pelo Programa Tesc, versão 3.04, (EMIC, Curitiba/PR, Brasil) como força máxima quando este fratura (Fig. 4).

O torque de fratura foi calculado multiplicando-se a força máxima pelo raio do eixo que o fio de polímero fora enrolado, conforme a equação:

Torque $(\mathrm{T})=$ Força $(\mathrm{F}) \times 4$,

Para os mini-implantes que fraturaram durante os ensaios de inserção ou remoção, utilizou-se o valor do torque no momento da fratura.

\section{Avaliação por microscopia}

A região de fratura dos mini-implantes foi avaliada através da microscopia eletrônica de varredura (MEV) com microscópio JEOL modelo JSM-5800 LV (JEOL, Tóquio, Japão). Uma vez que alguns mini-implantes fraturaram dentro do osso, apenas a parte superior poderia ser submetida ao MEV sem preparo específico. Desta forma, os fragmentos superiores dos mini-implantes foram colocados em placa metálica e presos por uma fita adesiva dupla-face, para permanecerem em posição vertical. Com o auxílio do programa específico do MEV, o mini-implante foi localizado e a região em que houve a deformação foi analisada e fotografada com aumento de 500x.
Com estas imagens, pôde-se determinar, por inspeção visual, o tipo de fratura para cada grupo de mini-implantes.

Para se verificar diferenças entre a resistência do material de confecção dos mini-implantes, utilizou-se a tensão cisalhante calculada, obtida através da seguinte fórmula:

$$
\text { Tensão cisalhante }=16 \cdot \mathrm{T} / \varpi \cdot \mathrm{D}^{3} \text {, }
$$

onde $\mathrm{T}=$ torque e $\mathrm{D}=$ diâmetro da superfície fraturada do mini-implante.

Para medir este diâmetro, utilizou-se o microscópio óptico Zeiss, Stemi 2000-C (Zeiss, Jena, Alemanha) com aumento de 150x. As imagens das superfícies foram capturadas em computador e enviadas ao programa Axio Vision (Zeiss, Jena, Alemanha), onde seus diâmetros foram calculados. Dois traços, perpendiculares entre si, contendo o diâmetro da superfície, foram marcados e a média destes dois valores foi considerada o diâmetro da superfície fraturada. Para alguns mini-implantes, estes valores foram confirmados no MEV (Fig. 5).

Para confirmar os valores da tensão cisalhante, calculou-se a tensão normal através da seguinte fórmula:

$$
\text { Tensão normal }=16 \cdot \mathrm{T} / \sqrt{3} \cdot \varpi \cdot \mathrm{D}^{3},
$$

onde $\mathrm{T}=$ torque e $\mathrm{D}=$ diâmetro da superfície fraturada do mini-implante.

\section{Análise estatística}

Todos os resultados numéricos foram apresentados através da média e do desvio-padrão. Os torques de inserção, de remoção e de fratura, assim como a tensão cisalhante calculada, foram comparados entre todos os grupos através do one-way ANOVA. Para se comparar os torques de inserção e de remoção para cada grupo, utilizou-se o twoway ANOVA. O nível de significância foi estabelecido em $\mathrm{p}<0,05$.

\section{RESULTADOS}

Os torques máximos de inserção dos mini-implantes na cortical de osso bovino foram de 25,2 $\pm 1,9 \mathrm{Ncm}, 23,2 \pm 4,9 \mathrm{Ncm}, 26,0 \pm 2,4 \mathrm{Ncm}$ e 30,6 


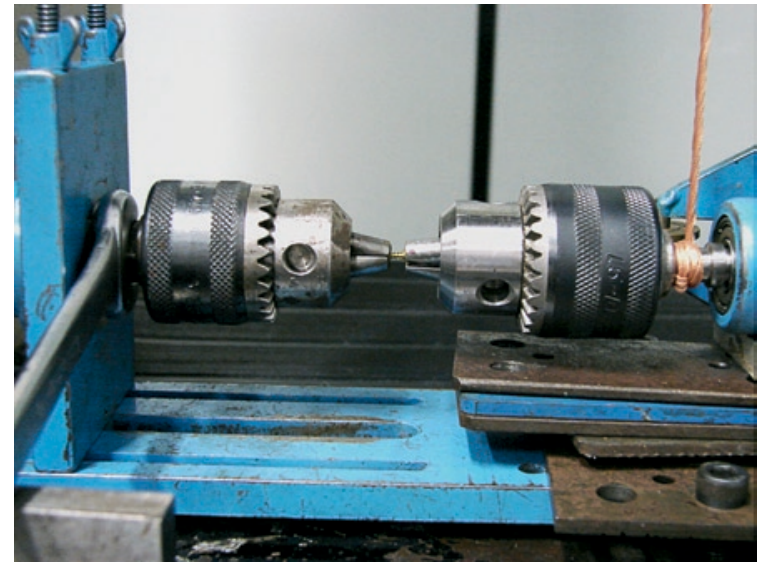

FIGURA 4 - Dispositivo de ensaio de torção, realizado para determinar o torque máximo de resistência à fratura dos mini-implantes, acoplado à máquina de ensaios mecânicos com célula de carga de $500 \mathrm{~N}$. 0 mandril da direita sofre rotação quando o fio de polímero é tracionado pela máquina de ensaio e 0 mandril da esquerda é fixo e prende o mini-implante.

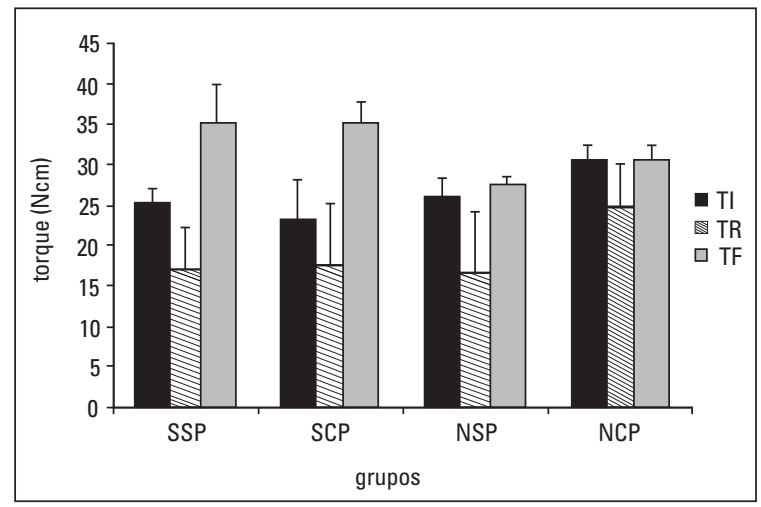

GRÁFICO 1 - Torques máximos ( $N c m$ ) de inserção (TI) e de remoção (TR) dos 4 grupos de mini-implantes. As colunas representam a média e as barras de erro representam o desvio-padrão. 0 número de amostras em cada grupo é de cinco, com exceção dos grupos NSP e NCP para os ensaios de remoção, que tiveram tamanho amostral de três.

$\pm 1,8 \mathrm{Ncm}$ para os grupos SSP, SCP, NSP e NCP, respectivamente (Gráf. 1). Dois mini-implantes do grupo NSP e dois do grupo NCP fraturaram durante o procedimento de inserção. Nesses casos, o valor de torque máximo registrado foi o obtido no momento da fratura. As médias dos torques de inserção entre os diferentes grupos de mini-implantes foram comparadas pela one-way ANOVA (Tab. 1). Verificou-se diferença estatisticamente

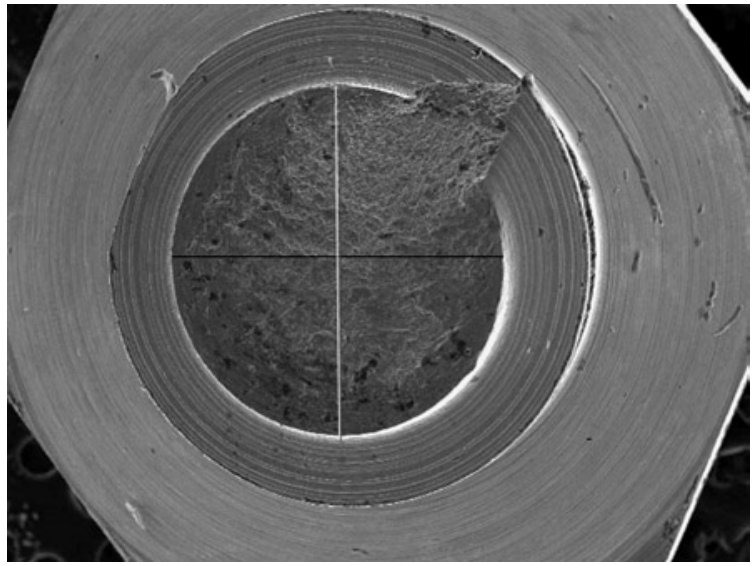

FIGURA 5 - Secção transversa do mini-implante fraturado observada no MEV em aumento de 500x. As linhas preta e branca foram utilizadas para se calcular a média do diâmetro no local de fratura e assim obter a tensão cisalhante calculada.

significativa para o grupo NCP, quando comparado com os demais grupos, demonstrando que o torque de inserção máximo do grupo NCP foi significativamente superior ao de todos os outros grupos.

Os torques máximos de remoção dos miniimplantes da cortical óssea também foram mensurados. As médias encontradas foram de 17,2 \pm $4,9 \mathrm{Ncm}, 17,6 \pm 7,6 \mathrm{Ncm}, 16,6 \pm 7,5 \mathrm{Ncm}$ e 25,0 $\pm 5,5 \mathrm{Ncm}$ para os grupos SSP, SCP, NSP e NCP, respectivamente (Gráf. 1). Três mini-implantes do grupo NCP fraturaram durante a remoção e o valor de torque máximo registrado também foi o obtido no momento da fratura. Os valores de torque máximo para inserção foram maiores do que os de remoção para todos os grupos, sendo que o grupo NCP apresentou os maiores valores de torque para estas duas variáveis. Não houve diferença significativa para o torque máximo de remoção entre os grupos (Tab. 1).

Para cada grupo, as médias dos torques máximos de inserção e de remoção foram comparadas entre si através da two-way ANOVA (Tab. 2). Apenas o grupo SSP apresentou diferença estatisticamente significativa, demonstrando que, para 
Tabela 1 - Comparação estatística (one-way ANOVA) entre as médias dos torques máximos de inserção, remoção e fratura de todos os grupos. Os números representam os valores de $\mathrm{p}$ e são assinalados com asterísco quando significativos.

\begin{tabular}{ccccccc|} 
& \multicolumn{6}{c}{ grupos } \\
& $\begin{array}{c}\text { SSP x } \\
\text { SCP }\end{array}$ & $\begin{array}{c}\text { SSP x } \\
\text { NSP }\end{array}$ & $\begin{array}{c}\text { SSP x } \\
\text { NCP }\end{array}$ & $\begin{array}{c}\text { SCP x } \\
\text { NSP }\end{array}$ & $\begin{array}{c}\text { SCP x } \\
\text { NCP }\end{array}$ & $\begin{array}{c}\text { NSP x } \\
\text { NCP }\end{array}$ \\
inserção & 0,421 & 0,574 & $0,001^{*}$ & 0,287 & $0,013^{*}$ & $0,009^{*}$ \\
remoção & 0,924 & 0,652 & 0,372 & 0,135 & 0,311 & 0,191 \\
fratura & 0,992 & $0,034^{*}$ & 0,160 & $0,003^{*}$ & $0,036^{*}$ & $0,003^{*}$
\end{tabular}

Tabela 2 - Comparação estatística (two-way ANOVA) entre as médias dos torques máximos de inserção e de remoção para os mini-implantes do mesmo grupo. Os números representam os valores de p e são assinalados com asterísco, quando significativos.

\begin{tabular}{|ccccc} 
& \multicolumn{5}{c}{ grupos } \\
& SSP & SCP & NSP & NCP \\
p & $0,044^{*}$ & 0,287 & 0,272 & 0,177 \\
\hline
\end{tabular}

este grupo, o torque máximo de inserção foi significativamente maior do que o de remoção, apesar de todos os outros grupos apresentarem o mesmo comportamento.

A média dos torques de fratura dos mini-implantes foi de $35,1 \pm 4,9 \mathrm{Ncm}, 35,1 \pm 2,7 \mathrm{Ncm}$, $27,4 \pm 1,1 \mathrm{Ncm}$ e $30,6 \pm 1,8 \mathrm{Ncm}$, para os grupos SSP, SCP, NSP e NCP, respectivamente (Gráf. 1). Os grupos SSP e SCP apresentaram valores mais próximos entre si do que os grupos NSP e NCP. Ao comparar os torques de fratura entre os grupos (Tab. 1), não se verificou diferença entre SSP e SCP e entre SSP e NCP. O grupo NSP apresentou a menor média para o torque de fratura e diferiu significativamente de todos os outros grupos. Os mini-implantes da marca SIN (grupos SSP e SCP) não apresentaram diferenças entre si, demonstrando pouca variação de resistência.

As fraturas das superfícies dos mini-implantes para todos os grupos foram comparadas por inspeção visual em MEV. Todos os grupos apresentaram

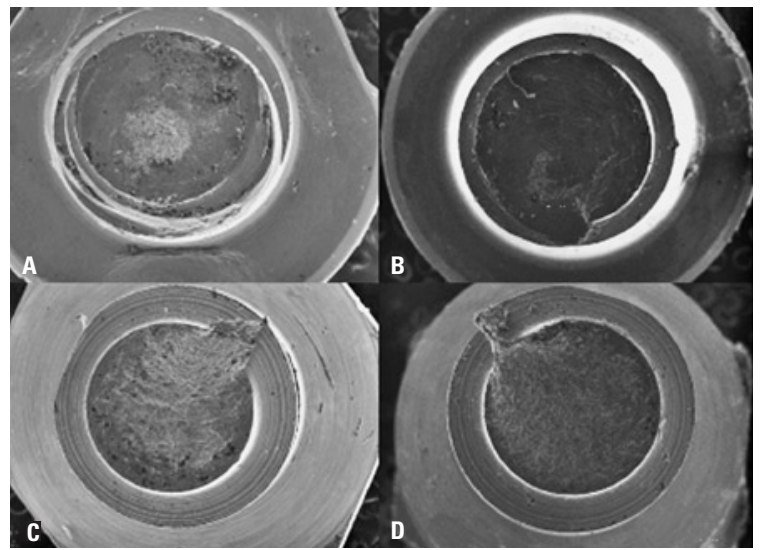

FIGURA 6 - Secção transversa de um mini-implante fraturado de cada grupo observada no MEV em aumento de 500x: A) mini-implante do grupo SSP; B) mini-implante do grupo SCP; $\mathbf{C}$ ) mini-implante do grupo NSP e D) mini-implante do grupo NCP. Estas imagens foram utilizadas para classificar o tipo de fratura ocorrido. Todas foram do tipo dúctil, caracterizado pelas linhas de cisalhamento geradas pela torção.

microporosidades e linhas de deformação plástica causadas por deformação em torção. As direções das linhas indicaram que as fraturas ocorreram por cisalhamento, caracterizando fraturas do tipo dúctil (Fig. 6).

A tensão cisalhante calculada foi de 1123,1 $\pm 168,3 \mathrm{MPa}, 1041,9 \pm 154,8 \mathrm{MPa}, 1124,8 \pm$ $123,0 \mathrm{MPa}$, e $1088,7 \pm 128,7 \mathrm{MPa}$ para os grupos SSP, SCP, NSP e NCP, respectivamente (Tab. 3). Não se verificou diferença estatisticamente significativa entre os grupos, quando comparados pela ANOVA, demonstrando que todos os miniimplantes não diferiram em relação à resistência mecânica do material com que foram confeccionados. Estes resultados também foram confirmados pela tensão normal calculada.

Para se calcular a tensão cisalhante foi necessário medir o diâmetro dos mini-implantes, sem contar a rosca, o que pode ser denominado de "alma" do mini-implante. Pôde-se perceber que o aumento do diâmetro de seção transversal da superfície de fratura foi acompanhado pelo aumento do torque (Tab. 3).

Em observação macroscópica dos mini-implantes utilizados neste estudo, observou-se diferenças nas suas características para os diferentes fabri- 
Tabela 3 - Valores do diâmetro (D) da região de fratura dos mini-implantes, da força para fratura (F), do torque de fratura $(T)$, da tensão cisalhante calculada (Tcis) e da tensão normal calculada (Tn). Os resultados são dados em média e desvio-padrão de 5 amostras em cada grupo.

\begin{tabular}{ccccc}
\multicolumn{5}{c}{ grupos } \\
& SSP & SCP & NSP & NCP \\
$\begin{array}{c}\text { D médio } \\
\text { (mm) }\end{array}$ & $1,2 \pm 0,0$ & $1,2 \pm 0,0$ & $1,1 \pm 0,0$ & $1,1 \pm 0,0$ \\
\hline $\mathbf{F}$ (N) & $88,0 \pm 12,2$ & $88,0 \pm 6,8$ & $69,18 \pm 2,25$ & $77,5 \pm 4,56$ \\
\hline $\begin{array}{c}\text { torque } \\
\text { (Ncm) }\end{array}$ & $35,1 \pm 4,9$ & $35,1 \pm 2,7$ & $27,4 \pm 1,1$ & $30,6 \pm 1,8$ \\
\hline $\begin{array}{c}\text { Tcis } \\
\text { (MPa) }\end{array}$ & $1123,1 \pm 168,3$ & $1041,9 \pm 154,8$ & $1124,8 \pm 123,0$ & $1088,7 \pm 128,7$ \\
$\begin{array}{c}\text { Tn } \\
\text { (MPa) }\end{array}$ & $648,5 \pm 97,1$ & $601,5 \pm 89,4$ & $634,0 \pm 70,6$ & $631,1 \pm 64,6$ \\
\hline
\end{tabular}

cantes, especialmente no número e na proximidade dos filetes das roscas (Fig. 1). Essa observação pôde ser comprovada em imagens feitas no MEV, demonstrando que os mini-implantes dos grupos NSP e NCP apresentaram maior número e proximidade de filetes de roscas, quando comparados aos grupos SSP e SCP (Fig. 7).

\section{DISCUSSÃO}

As dimensões reduzidas dos mini-implantes permitem sua inserção em diversas áreas na cavidade bucal, mas aumentam a possibilidade de deformação durante o emprego e a fratura durante a inserção ou remoção ${ }^{9}$. Neste estudo, miniimplantes ortodônticos foram analisados quanto à sua resistência, durante a inserção e remoção em cortical de osso bovino, e ao serem submetidos à torção até a sua fratura.

Foram escolhidos mini-implantes de duas das principais empresas nacionais, que possuíam as dimensões mais semelhantes. Todos os mini-implantes apresentavam o mesmo diâmetro, de $1,6 \mathrm{~mm}$, que foi considerada uma dimensão adequada para ser usada em todas as áreas indicadas na cavidade bucal $^{23}$. Além disso, a escolha por um diâmetro maior teve como objetivo obter valores de torque bastante elevados. Foi verificado, por Elias et al. ${ }^{9}$,

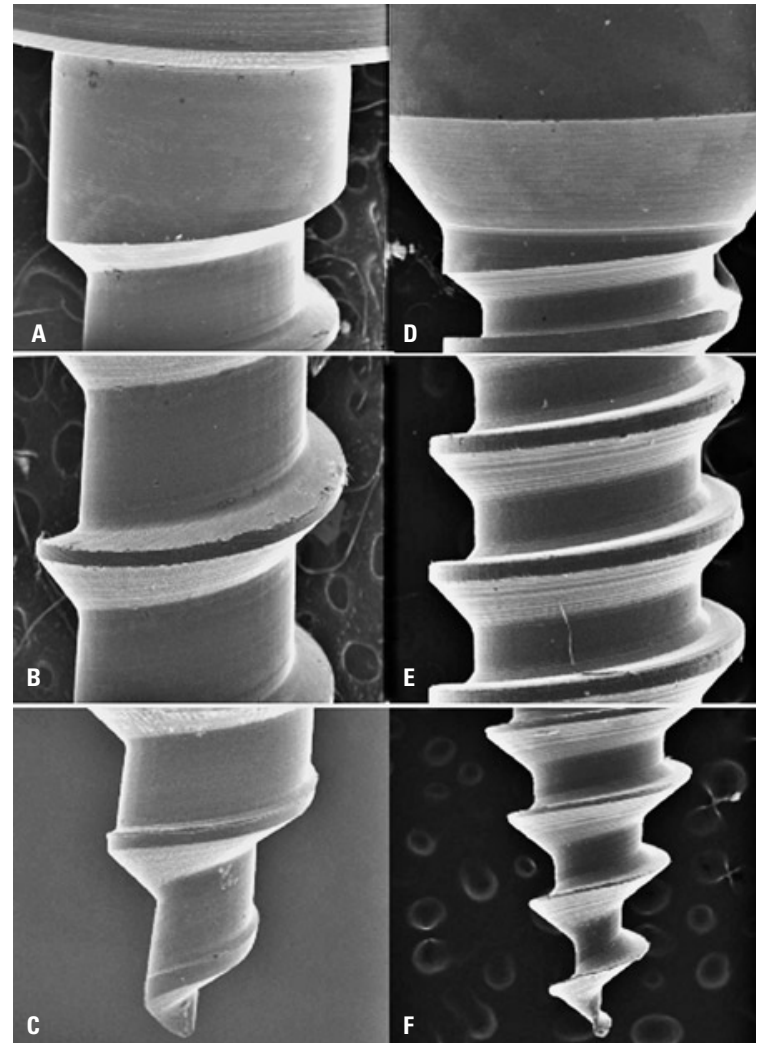

FIGURA 7 - Perfil de mini-implantes com 1,6mm de diâmetro observados no MEV em aumento de 500x. As figuras A, B e C correspondem ao mini-implante da SIN e as figuras D, E e F ao mini-implante da Neodent, sendo, respectivamente, a parte superior, média e a ponta ativa do corpo do mini-implante. Todas as áreas apresentam dimensões diferentes, apesar dos mini-implantes terem as mesmas especificações comerciais. 0 número de filetes de roscas, a distância entre os filetes e as pontas ativas são diferentes.

que, comparando-se dois tipos de mini-implantes do mesmo fabricante com diâmetros diferentes, quanto maior o diâmetro do mini-implante maior o seu torque de inserção, já que este é proporcional à área de contato do mini-implante com o osso.

A presença ou ausência de perfil transmucoso foi uma das variáveis analisadas no presente trabalho. O objetivo dessa estrutura é manter a saúde dos tecidos peri-implantares, principalmente em áreas com pequena faixa de gengiva inserida, pois a ausência de inflamação é fator que contribui para a melhor estabilidade do mini-implante ${ }^{3}$. Foram escolhidos mini-implantes sem e com perfil transmucoso de $2 \mathrm{~mm}$ para os dois fabricantes, 
isto é, com a maior variação possível, pois este é o maior perfil transmucoso fabricado pelas empresas Neodent e SIN.

O comprimento escolhido foi o mais próximo entre as empresas, $7 \mathrm{~mm}$ para o da Neodent e $8 \mathrm{~mm}$ para o da SIN, pois as duas empresas não fabricam mini-implantes com os mesmos comprimentos.

O sucesso da utilização de mini-implantes ortodônticos está relacionado à sua estabilidade primária após a instalação. A estabilidade primária depende, principalmente, da forma do implante e da qualidade óssea do local de inserção. $\mathrm{O}$ apoio em osso cortical é essencial para que haja estabilidade primária, pois a pequena espessura desse tipo de osso resulta em falha na utilização de mini-implantes ${ }^{3}$. Desta forma, a tíbia bovina foi escolhida para os ensaios, devido à quantidade de cortical encontrada nesse osso, permitindo a inserção completa da parte ativa do mini-implante.

Os valores médios para o torque máximo de inserção obtidos neste estudo variaram de 30,6 a $23,2 \mathrm{Ncm}$. Tais valores são compatíveis com os resultados encontrados por Wilmes et al. ${ }^{25}$, que variaram de 41,3 a 23,4 $\mathrm{Ncm}$, mesmo tendo sido inseridos em ossos de pelve de porcos com cortical óssea muito menos espessa do que a utilizada neste estudo. Motoyoshi et al. obtiveram valores de torques para inserção muito mais baixos do que os observados neste estudo, que variaram de 7,2 a $13,5 \mathrm{Ncm}$ em adultos ${ }^{17}$ e 7,6 a $9,2 \mathrm{Ncm}$ em adolescentes $^{18}$. Elias et al. ${ }^{9}$ obtiveram, para miniimplantes de $1,5 \mathrm{~mm}$, torque médio de inserção de $9,6 \mathrm{Ncm}$ em cortical de coelhos e $12,6 \mathrm{Ncm}$ em cortical bovina, sendo também valores muito inferiores aos encontrados neste estudo. Os miniimplantes com 2,0mm de diâmetro, quando inseridos em cortical bovina, apresentaram torque médio de inserção de $23,2 \mathrm{Ncm}$, mais próximo aos valores obtidos neste estudo.

Os valores médios para o torque máximo de remoção obtidos neste estudo variaram de 25,0 (grupo NCP) a 16,6Ncm (grupo NSP) e não houve diferença significativa entre os grupos. Elias et al. ${ }^{9}$ avaliaram o torque de remoção de mini-implantes comerciais com $6,0 \mathrm{~mm}$ de comprimento e diâmetros de 1,5 e 2,0mm e encontraram valores de $5,4 \pm 0,7 \mathrm{Ncm}$ para a cortical de coelho e $6,8 \pm$ $0,8 \mathrm{Ncm}$ na cortical bovina, para os mini-implantes de $1,5 \mathrm{~mm}$ de diâmetro. Os mini-implantes com 2,0mm de diâmetro só foram testados em cortical bovina e apresentaram torque de remoção de $12,0 \pm 1,6 \mathrm{Ncm}$. Estes valores foram inferiores aos encontrados neste estudo, mesmo para os mini-implantes com maior diâmetro. Contudo, os mini-implantes utilizados por Elias et al..$^{9}$ eram mais curtos (6mm) e não foram inseridos apenas em cortical óssea.

Assim como no torque de inserção, o grupo NCP obteve os maiores valores de torque de remoção, o que mostra que também houve maior dificuldade em removê-los do osso. Apenas a presença ou ausência de perfil transmucoso parece não afetar os torques de inserção ou remoção, uma vez que só o grupo NCP apresentou valores significativos, enquanto o grupo SCP não apresentou diferenças.

Ao comparar valores de torque de inserção e de remoção em mini-implantes, Elias et al. ${ }^{9}$ observaram que o torque de remoção é menor do que o torque de inserção, independente do tipo de cortical ou do diâmetro dos mini-implantes, o que também foi observado neste estudo. Porém, apenas o grupo SSP apresentou diferença significativa entre a média de torque de inserção e a média de torque de remoção. Dilek et al. ${ }^{8}$ relataram torques de remoção mais elevados que os de inserção, em ensaio não-vital em fêmur bovino, discordando dos outros estudos. Torques mais elevados de remoção do que de inserção são encontrados em estudos in vivo, quando há um acompanhamento de, pelo menos, 4 semanas, permitindo a osteointegração do dispositivo $5,6,16,17,18$.

Durante os ensaios de inserção, dois mini-implantes dos grupos NSP e NCP fraturaram e os outros três do grupo NCP fraturaram durante os ensaios de remoção. Nenhum mini-implante dos 
grupos SSP e SCP fraturou nestes ensaios. Os grupos NSP e NCP apresentaram os maiores torques de inserção, o que pode explicar as fraturas. A presença do perfil transmucoso parece não afetar o valor dos torques para os grupos da SIN, mas parece existir diferença entre os da Neodent. $\mathrm{O}$ fato de mais mini-implantes terem fraturado durante os ensaios de remoção corrobora os achados de Melsen ${ }^{15}$, que afirma ser este o momento de maior ocorrência de fraturas de mini-implantes.

Os torques de fratura variaram entre 35,14 (grupo SSP) e 27,42Ncm (grupo NSP). Os grupos SSP e SCP apresentaram torques de fratura muito próximos, enquanto os grupos NSP e NCP apresentaram valores muito discrepantes. Realizou-se, também, a comparação do torque de fratura entre os grupos. Observou-se que, entre os mini-implantes da SIN, não houve diferença significativa, no entanto, os mini-implantes da Neodent apresentaram diferença entre si. Dilek et al. ${ }^{8}$ relatam que torques entre 35 e $50 \mathrm{Ncm}$ podem causar fratura do mini-implante. Wilmes ${ }^{25}$ recomenda limitar o torque de inserção a $20 \mathrm{Ncm}$, para evitar as fraturas.

Diante dos resultados, fica evidente haver diferenças entre os mini-implantes de fabricantes diferentes. Para verificar se a resistência mecânica do material de fabricação era semelhante, os miniimplantes foram submetidos à avaliação microscópica das suas superfícies fraturadas. Observou-se que todos os grupos apresentaram fratura do tipo dúctil, ou seja, com deformação plástica. Essa característica mostra que, provavelmente, todos os mini-implantes estudados são compatíveis quanto ao material.

A tensão cisalhante calculada e a tensão normal calculada, obtidas no momento da fratura, permitem verificar se o material de fabricação do mini-implante é semelhante e representam o comportamento mecânico do material. Para se calcular estas tensões, mediu-se o diâmetro da região de fratura do mini-implante. Os valores encontrados de tensão cisalhante e normal não tiveram diferença estatística significativa entre os grupos e, portanto, não foi observada diferença na resistência mecânica entre os materiais utilizados pelos diferentes mini-implantes. Uma vez que não foi determinada diferença na resistência mecânica dos mini-implantes, nem na morfologia da superfície de fratura, as diferenças na resistência ao torque observadas podem estar relacionadas à forma do mini-implante.

Pôde-se perceber que o aumento do diâmetro de seção transversal da superfície de fratura foi acompanhado pelo aumento do torque de fratura. O diâmetro apresentado pelo fabricante é o diâmetro total, sendo a informação clínica necessária para se saber o quanto de espaço o dispositivo irá ocupar. Mas, através destes resultados, verificou-se que existe diferença no diâmetro da "alma" nos diferentes grupos avaliados. Sendo assim, a forma do mini-implante é uma variável que deve ser considerada ao se avaliar a resistência mecânica deste produto. Verificou-se, também, um maior número e maior proximidade nos filetes de roscas dos grupos NSP e NCP, o que pode gerar maior imbricamento mecânico e, conseqüentemente, maior resistência na inserção do mini-implante no osso. O menor diâmetro da "alma" e os maiores torques de inserção podem explicar a menor resistência a fraturas dos mini-implantes destes grupos.

Com a avaliação dos resultados obtidos através da análise mecânica dos mini-implantes, percebese que há necessidade de padronização de todas as estruturas deste produto, isto é, diâmetro da "alma", forma do mini-implante, tamanho do mini-implante, e forma e proximidade dos filetes das roscas. Fraturas de mini-implantes durante a inserção ou na aplicação de força ortodôntica podem ser um problema grave, impedindo até mesmo a futura movimentação dentária ${ }^{14}$. A literatura atual possui muita informação clínica, no entanto há pouca associação do que se sabe sobre as características ideais da morfologia dos mini-implantes com suas aplicações clínicas. Com a crescente utilização deste dispositivo, sugere-se que novas pes- 
quisas sejam feitas, buscando melhorar e adaptar a forma dos mini-implantes à sua melhor utilização nas diversas aplicações clínicas que são propostas no tratamento ortodôntico.

\section{CONCLUSÃO}

O grupo NCP apresentou o maior torque de inserção e foi significativamente diferente de todos os outros grupos. O torque de remoção dos mini-implantes não apresentou diferença estatisticamente significativa entre os grupos, mas foi sempre menor do que os torques de inserção. $\mathrm{O}$ grupo NCP diferiu significativamente de todos os outros grupos, apresentando a menor resistência à fratura. Os mini-implantes da marca SIN (grupos SSP e SCP) não apresentaram diferença entre si, demonstrando pouca variação de resistência. Todos os grupos apresentaram fratura do tipo dúctil, em inspeção por microscopia eletrônica de varredura, demonstrando haver compatibilidade no material de confecção dos mini-implantes, apesar de serem de fabricantes diferentes. Isso foi confirmado pela ausência de diferença na tensão cisalhante máxima calculada entre os grupos.

Os mini-implantes da Neodent apresentaram, em geral, comportamento diferente dos da SIN. Uma vez que os mini-implantes são confeccionados com o mesmo material, pode-se dizer que a diferença na forma, diâmetro da "alma" e número de filetes de roscas pode afetar as características físicas do dispositivo, notadamente os torques de inserção, de remoção e de fratura.

\title{
Evaluation of insertion, removal and fracture torques of different orthodontic mini- implants in bovine tibia cortex
}

\begin{abstract}
Aim: The purpose of this study was to evaluate mini-implants with different dimensions for the following factors: (a) insertion torque, (b) removal torque, (c) fracture torque, (d) shear tension, (e) normal tension and ( $f$ ) type of fracture. Methods: Twenty self-drilling mini-implants were used, 10 from SIN and 10 from Neodent, with $1.6 \mathrm{~mm}$ in diameter and with 8 and $7 \mathrm{~mm}$ in length, respectively. Out of these 10 mini-implants, 5 did not have a neck and the other 5 had a $2 \mathrm{~mm}$ neck, and were separated into 4 groups: SIN without neck (SSP), SIN with neck (SCP), Neodent without neck (NSP) and Neodent with neck (NCP). All mini-implants were inserted in bone cortex and removed with a low speed handpiece connected to a digital torquimeter. The mini-implants were also submitted to a fracture test. The insertion, removal and fracture torques, as well as the calculated shear and normal tensions, were compared between all groups by ANOVA. The type of fracture was assessed by a scanning electronic microscope. Results: The NCP group presented a significantly greater insertion torque than all other groups, but all of them fractured during insertion $(n=2)$ or removal $(n=3)$. There were no significant differences among groups for removal torque. For group NSP, the fracture torque was significantly smaller than all other groups. All mini-implants suffered ductile fracture. Conclusion: Since there were no differences for the mechanical resistance of both brands of mini-implants, varying only in shape, one may conclude that resistance to fracture can be affected by this variable.
\end{abstract}

Key words: Dental implants. Material resistance. Torque. Orthodontic anchorage procedures. 


\section{REFERÊNCIAS}

1. ANGLE, E. H. Malocclusion of the teeth. 7th ed. Philadelphia: S. S. White Dental Manufacturing, 1907.

2. ARAÚJO, T. M.; NASCIMENTO, M. H. A.; BEZERRA, F.; SOBRAL, M. C. Ancoragem esquelética em Ortodontia com mini-implantes. Rev. Dental Press Ortodon. Ortop. Facial Maringá, v. 11, n. 4, p. 126-156, jul./ago. 2006.

3. ARAÚJO, T. M. Ancoragem esquelética com mini-implantes. In: LIMA FILHO, R. M. A.; BOLOGNESE, A. M. Ortodontia: arte e ciência. 1. ed. Maringá: Dental Press, 2007. cap. 19, p. 393-448.

4. BAE, S. M.; PARK, H. S.; KYUNG, H. M.; KWON, O. W.; SUNG, J. H. Clinical application of micro-implant anchorage. J. Clin. Orthod., Boulder, v. 36, no. 5, p. 298-302, May 2002.

5. BÜCHTER, A.; WIECHMANN, D.; KOERDT, S.; WIESMANN, H. P.; PIFFKO, J.; MEYER, U. Load-related implant reaction of miniimplants used for orthodontic anchorage. Clin. Oral Implants Res., Copenhagen, v. 16, no. 4, p. 473-479, Aug. 2005.

6. CHEN, Y.; SHIN, H. I.; KYUNG, H. M. Biomechanical and histological comparison of self-drilling and self-tapping orthodontic microimplants in dogs. Am. J. Orthod. Dentofacial Orthop., St. Louis, v. 133, no. 1, p. 44-50, Jan. 2008

7. COSTA, A.; RAFFAINI, M.; MELSEN, B. Miniscrews as orthodontic anchorage: a preliminary report. Int. J. Adult Orthodon. Orthognath. Surg., Chicago, v. 13, no. 3 , p. 201-219, 1998

8. DILEK, O.; TEZULAS, E.; DINCEL, M. Required minimum primary stability and torque values for immediate loading of mini dental implants: an experimental study in non-viable bovine femoral bone. Oral Surg. Oral Med. Oral Pathol. Oral Radiol. Endod., St. Louis, v. 105, no. 2 , p. 20-27, Feb. 2007.

9. ELIAS, C. N.; GUIMARÃES, G. S.; MULLER, C. A. Torque de inserção e de remoção de miniparafusos ortodônticos. Rev. Bras. Implant., Rio de Janeiro, v. 11, n. 3, p. 5-8, 2005.

10. FAVERO, L.; BROLLO, P.; BRESSAN, E. Orthodontic anchorage with specific fixture: related study analysis. Am. J. Orthod. Dentofacial Orthop., St. Louis, v. 122, no. 1, p. 84-94, 2002.

11. FRIBERG, B.; SENNERBY, L.; GRONDAHL, K.; BERGSTROM, C.; BACK, T.; LEKHOLM, U. Identification of bone quality in conjunction with insertion of titanium implants: a pilot study in jaw autopsy specimens. Clin. Oral Implants Res., Copenhagen, v. 6, no. 4, p. 213-219, Dec. 1995

12. KANOMI, R. Mini-implants for orthodontic anchorage. J. Clin. Orthod., Boulder, v. 31, no. 11, p. 763-767, Nov. 1997.

13. KLOEHN, S. J. Evaluation of cervical anchorage force in treatment. Angle Orthod., Appleton, v. 31, no. 2, p. 91-104, Apr. 1961.
14. LABOISSIĖRE JÚNIOR, M.; VILLELA, H.; BEZERRA, F.; LABOISSIÈRE, M.; DIAZ, L. Ancoragem absoluta utilizando microparafusos ortodônticos: protocolo para aplicação clínica (Trilogia - Parte II). Implant News, São Paulo, v. 2, n. 1, p. 37-46, jan./fev. 2005.

15. MELSEN, B. Mini-implants: where are we? J. Clin. Orthod., Boulder, v. 39 , no. 9 p. 539-547, Sept. 2005.

16. MORAIS, L. S.; SERRA, G. G.; MULLER, C. A.; ANDRADE, L. R. PALERMO, E. F. A.; ELIAS, C. N.; MEYERS, M. Titanium alloy mini-implants for orthodontic anchorage: immediate loading and metal ion release. Acta Biomater., Kidlington, v. 3, no. 3, p. 331-339, May 2007.

17. MOTOYOSHI, M.; HIRABAYASHI, M.; UEMURA, M.; SHIMIZU, $\mathrm{N}$. Recommended placement torque when tightening an orthodontic mini-implant. Clin. Oral Impl. Res., Copenhagen, v. 17, no. 1, p. 109-114, Feb. 2006.

18. MOTOYOSHI, M.; MASUOKA, M.; SHIMIZU, N. Application of orthodontic mini-implants in adolescents. Int. J. Oral Maxillofac. Surg., Copenhagen, v. 36, no. 8, p. 695-699, Aug. 2007.

19. OHMAE, M.; SAITO, S.; MOROHASHI, T.; SEKI, K.; QU, $H_{\text {.; }}$ KANOMI, R.; YAMASAKI, K.; OKANO, T.; YAMADA, S. SHIBASAKI, Y. A clinical and histological evaluation of titanium mini-implants as anchors for orthodontic intrusion in the beagle dog. Am. J. Orthod. Dentofacial Orthop., St. Louis, v. 119, no. 5, p. 489-497, May 2001

20. PROFFIT, W. R.; FIELDS, H. W. Contemporary Orthodontics. 3rd ed. St. Louis: CV Mosby, 1999.

21. ROBERTS, W. E.; SMITH, R. K.; ZILBERMAN, Y.; MOZSARY, P. G.; SMITH, R. S. Osseous adaptation to continuous loading of rigid endosseous implants. Am. J. Orthod., St. Louis, v. 86, no. 2, p. 95-111, Aug. 1984.

22. SHAPIRO, P. A.; KOKICH, V. G. Uses of implants in Orthodontics. Dent. Clin. North Am., Philadelphia, v. 32, no. 3, p. 539-550, 1988.

23. SUNG, J. H.; KYUNG, H. M.; BAE, S. M.; PARK, H. S.; KWON, O. W.; McNAMARA JUNIOR, J. A. Mini-implantes. 1. ed. Nova Odessa: Ed. Napoleão, 2007.

24. THUROW, R. C. Craniomaxillary orthopedic correction with en masse dental control. Am. J. Orthod., St. Louis, v. 68, no. 6, p. 601-624, Dec. 1975.

25. WILMES, B.; RADEMACHER, C.; OLTHOFF, G.; DRESCHER, D. Parameters affecting primary stability of orthodontic miniimplants. J. Orofac. Orthop., München, v. 67, no. 3, p. 162-174, May 2006
Endereço para correspondência

Flavia Artese

Rua Santa Clara, 75/1110 - Copacabana

CEP: 22.041-010 - Rio de Janeiro

E-mail: flaviaartese@gmail.com 